\title{
Evidencia de sobrediagnóstico en el TDAH en base a evaluación neuropsicológica: un estudio en escolares chilenos
} \section{(4) PSicogenTE}
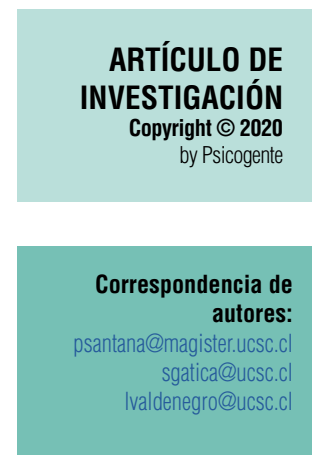

Recibido: 03-09-2019 Aceptado: $18-03-2020$ Publicado: 05-06-20

\section{Evidence of overdiagnosis in Attention Deficit Hyperactivity Disorder (ADHD) based on neuropsychological evaluation: a study in Chilean students}

\author{
Paulina Isabel Santana-Vidal (D) - Sergio Andrés Gatica-Ferrero (iD) \\ Lidia Verónica Valdenegro-Fuentes (iD) \\ Universidad Católica de la Santísima Concepción, Concepción, Chile
}

\section{Resumen}

Objetivo: El Trastorno por Déficit de Atención con Hiperactividad/impulsividad (TDAH) es una de las dificultades de aprendizaje más comunes en las escuelas chilenas. Se caracteriza por un patrón de funcionamiento atípico en atención e inhibición, con fuerte compromiso de otras funciones ejecutivas. Uno de los desafíos que plantea este trastorno está referido al proceso de diagnosis, el cual no suele ser suficientemente preciso. Se pretende explorar las diferencias en la distribución de dos muestras de estudiantes con y sin TDAH diagnosticadas con una escala de observación conductual a través de la evaluación de las funciones ejecutivas de atención, inhibición y flexibilidad cognitiva.

Método: En este estudio se evaluó a 132 escolares, 66 con TDAH y 66 sin TDAH, en las variables de atención, inhibición y flexibilidad cognitiva de un colegio de la ciudad de Concepción en Chile. Se contrastó el rendimiento cognitivo con los diagnósticos y se reagrupó a los estudiantes en los subtipos conocidos del trastorno.

Resultados: La evaluación neuropsicológica mostró que en el grupo con TDAH había alumnos que no cumplían los criterios diagnósticos para estar incluidos en él. En el grupo sin TDAH se observó un fenómeno semejante. En ambos grupos la evaluación neuropsicológica de la atención y la inhibición resultaron útiles para diagnosticar con mayor certeza y para determinar el subtipo al que pertenecía cada estudiante con TDAH detectado. La flexibilidad cognitiva solo permitió diferenciar a los sujetos con $y \sin$ TDAH.

Conclusiones: En ambos grupos estudiados fue posible encontrar sujetos mal diagnosticados; el sobrediagnóstico fue de $43,93 \%$ en el grupo con TDAH, mientras que el infradiagnóstico fue de $42,42 \%$ en el grupo $\sin$ TDAH.

Palabras clave: trastorno de Déficit de Atención con Hiperactividad, TDAH, sobrediagnóstico, infra diagnóstico, evaluación neuropsicológica, dificultades de aprendizaje, funciones ejecutivas, atención, inhibición.

\section{Abstract}

Objective: Attention Deficit Hyperactivity/Impulsivity Disorder (ADHD) is one of the most common learning difficulties in Chilean schools. It is characterized by an atypical functioning pattern in attention and inhibition, strongly compromising other executive functions. One of the challenges posed by this disorder is the diagnosis process, which is often not sufficiently accurate. The intention is to study the distribution of two students samples, with and without ADHD, diagnosed by a behavioral observation scale through the evaluation of attention, inhibition, and cognitive flexibility executive functions.

Method: This study evaluated 132 students, 66 with ADHD and 66 without ADHD, considering the 
attention, inhibition, and cognitive flexibility variables of a school in the city of Concepción in Chile. Cognitive performance was contrasted with the diagnoses, and students were regrouped in the disorder known subtypes.

Results: The neuropsychological evaluation showed that in the ADHD group, there were students who did not meet the diagnostic criteria to be included in the group. A similar phenomenon was observed in the group without ADHD. In both the groups, the neuropsychological evaluation of attention and inhibition was useful in providing a more certain dignosis and in determining the subtype to which each student detected with ADHD belonged. Cognitive flexibility was only useful in differentiating betwen the subjects with and without ADHD.

Conclusions: In both the groups studied, it was possible to find misdiagnosed subjects; the overdiagnosis was $43,93 \%$ in the ADHD group, while the under-diagnosis was $42,42 \%$ in the group without ADHD.

Keywords: Attention Deficit Hyperactivity Disorder, ADHD, overdiagnosis, under-diagnosis, neuropsychological evaluation, learning difficulties, executive functions, attention, inhibition.

\section{INTRODUCCIÓN}

El Trastorno por Déficit de Atención con Hiperactividad (TDAH) es uno de los diagnósticos más frecuentes en niños y adolescentes escolarizados. El TDAH es un trastorno de origen neurobiológico cuyas manifestaciones cognitivas y conductuales afectan la adaptación positiva a la escuela. EI TDAH tiene su inicio en la infancia y se caracteriza por un comportamiento persistente de inatención y/o hiperactividad-impulsividad (APA, 2014). Sus manifestaciones deben presentarse antes de los doce años de edad, interfiriendo notoriamente en el funcionamiento social, académico o laboral de la persona; la sintomatología TDAH no puede ser atribuible a alteraciones neurológicas, sensoriales, del lenguaje o motoras graves (APA, 2014). Los estudiantes con este trastorno tienen un mayor riesgo de fracaso escolar, problemas de comportamiento y dificultades en las relaciones socio-familiares (GarcíaNoriega \& Bárcena, 2013).

Los síntomas del TDAH pueden no ser evidentes en un entorno con sistemático feedback externo, en situaciones que impliquen actividades novedosas o donde las recompensas sean inmediatas. Por el contrario, la sintomatología se agrava o se hace visible ante un entorno no estructurado, en presencia de muchas distracciones o si se requiere de un esfuerzo mental sostenido. La variabilidad de escenarios que puede enfrentar el estudiante con TDAH hace necesario que la evaluación se realice en varias oportunidades y en diversos contextos (García-Noriega \& Bárcena, 2013). La prevalencia del TDAH ha resultado polémica; en USA un estudio en dos Estados la ha situado en el $8,7 \%$ y el $10,6 \%$, respectivamente (Wolraich et al., 2014). La APA (2014) la sitúa en torno al $5 \%$. En España, de acuerdo a los criterios del CIE-10 la prevalencia en escolares de 6 a 12 años es de 1,2 \% (Cardo, Servera, Vidal, de Azúa, Redondo \& Ruitort, 2011). En Gran Bretaña se informó una prevalencia de 7,4 \% en niños y adolescentes de 13 a 17 años (McCarthy, Wilton, Murray, 
Hodgkins, Asherson \& Wong, 2012). Un estudio basado en datos del Instituto de Salud Pública de Eslovenia sitúa la prevalencia del TDAH cerca del $1 \%$ (Štuhec, Švab \& Locatelli, 2015). En Chile la prevalencia ha sido situada entre el 7 \% en adultos jóvenes (Santander, Berner, Contreras \& Gómez, 2013) y $10 \%$ en niños, y en adolescentes el 10 \% (De la Barra, Vicente, Saldivia \& Melipillán, 2013). Estas discrepancias ha llevado a algunos autores a estimar que la prevalencia varía en función a la edad y la cantidad de síntomas observados por los informantes (McKeown, Holbrook, Danielson, Cuffe, Wolraich \& Visser, 2015).

López, Rodillo \& Kleinsteuber (2008) postulan que el diagnóstico del TDAH es descriptivo y se basa en la identificación de los criterios del sistema europeo de Clasificación Internacional de Enfermedades (CIE-11) y el DSM-V, clasificando a los sujetos que lo presentan en alguna de las tres manifestaciones clínicas conocidas, esto es TDAH con predominio inatento (TDAH-I), con predominio hiperactivo/impulsivo (TDAH-HI) o combinado (TDAH-C).

Barkley (2009) observó ciertos problemas en la aplicación clínica de los criterios del DSM-IV; específicamente señaló dificultades con la extensión del listado de síntomas y con sus definiciones operacionales. Del mismo modo, estimó que las observaciones respecto a estas cuestiones podrían mejorar los criterios diagnósticos de cara a la publicación del DSM-V, cuestión que finalmente no ocurrió. Otros autores (Abad-Mas, Caloca-Català, Mulas \& Ruiz-Andrés, 2017) concuerdan respecto a la extensión e imprecisión de la lista de síntomas, y plantean la necesidad de evaluar neuropsicológicamente los síntomas nucleares del trastorno a través de test que midan la atención, control inhibitorio y otras funciones ejecutivas. Existe evidencia que muestra que los estudiantes con TDAH presentan un bajo rendimiento en tareas cognitivas que implican habilidades de vigilancia, aprendizaje verbal, memoria de trabajo, flexibilidad cognitiva, planificación, resolución de problemas e inhibición de respuestas (Mulas et al., 2006; Abad-Mas, Caloca-Català, Mulas \& Ruiz-Andrés, 2017).

En la actualidad el diagnóstico de TDAH se realiza en base a la observación conductual y a la evaluación neuropsicológica; si bien existe una perspectiva que favorece la evaluación neuropsicológica por sobre la evaluación conductual (Etchepareborda, Paiva-Barón \& Abad, 2009; Tirapu-Ustárroz, García-Molina, Luna-Lario \& Periáñez, 2012; López-Soler \& Romero-Medina, 2013), algunos autores utilizan con éxito en el ámbito de la investigación las escalas de observación conductual como la Vanderbilt ADHD Rating Scale (Langberg, Vaughn, Brinkman, Froehlich \& Epstein, 2010; Becker, 2013; SalasBravo, González-Arias, Araya-Piñones, Valencia-Jiménez \& OyarceCortes, 
2017). Otros autores sostienen que ambas perspectivas pueden ser complementarias (Carreño-Álvarez \& Gatica-Ferrero, 2019).

Algunos de los instrumentos de observación conductual utilizados para la detección del TDAH, tanto en la práctica clínica como escolar, suelen ser el Cuestionario de Conducta de Conners para Profesores (Decreto 170/2010; Sims, Purpura \& Lonigan, 2012), el cuestionario ADHD Rating Scale IV (Langberg, Vaughn, Brinkman, Froehlich, \& Epstein, 2010; Becker, 2013), el Cuestionario para Escolares y Adolescentes Latinoamericanos con TDAH (CEAL-TDAH) (Ulloa-Flores, et al., 2009) y la escala NICHQ Vanderbilt Diagnostic Teacher Rating Scale (Langberg, Vaughn, Brinkman, Froehlich \& Epstein, 2010; Becker, 2013).

En Chile, los cuestionarios de observación conductual tienen un uso más habitual que la evaluación neuropsicológicas. Esta situación es discordante con la investigación actual, que plantea que la evaluación neuropsicológica cumple un rol complementario al uso de cuestionarios y de los criterios diagnósticos establecidos en el DSM-V (Abad-Mas, Caloca-Català, Mulas y Ruiz-Andrés, 2017). La aplicación de la Figura de Rey-Osterrieth y la prueba de Cancelación Visual de Mesalum, por un lado, y la cumplimentación de la encuesta de Conners para profesores no arrojó relaciones significativas (Flores-Aréchiga, Garza-González, Llaca-Díaz y Gómez-Espinel, 2016). Resultados semejantes se obtuvieron tras la aplicación de Letter Cancelation Test (LCT), Continuos Performance Test (CPT) y Matching Familiar Figures test (MFF), y el Young ADHD Questionnaire-I (YAQ-I), cuestionario autoinformado, y Young ADHD Questionnaire-S (YAQS), cuestionario informado por terceros, aplicado por examinadores entrenados; los resultados mostraron la ausencia de correlación estadística entre ambos procedimientos, aunque el uso exclusivo de test neuropsicológicos es insuficiente para discriminar entre el TDAH y algunos trastornos psiquiátricos (Young \& Gudjonsson, 2005).

Si bien no existe acuerdo respecto al perfil neuropsicológico de las personas con TDAH, hay un cierto consenso relativo a que las funciones ejecutivas alteradas en este trastorno serían la atención e inhibición como síntomas nucleares. Algunos autores han mencionado además, la flexibilidad cognitiva y la memoria de trabajo (Rubiales, 2012). Diversos trabajos confirman que los sujetos con TDAH presentan dificultades en tareas relacionadas con el control ejecutivo (Barkley, 1997a, 1997b; Nigg, 2001; Sergeant, 2005). La alteración de las funciones ejecutivas explicaría la variabilidad en las tres presentaciones clínicas del trastorno, esto es, predominio de inatento, predominio hiperactivo-impulsivo, y combinado (García, González-Castro, Rodríguez, Cueli, 
Álvarez \& Álvarez, 2014); el déficit ejecutivo descrito refuerza la descripción conductual del trastorno que se realiza a través de las escalas de observación.

La atención ha sido una función ejecutiva bastante estudiada en el caso del TDAH, y se le ha descrito como un mecanismo cognitivo que permite mantener el estado de vigilancia ante determinados estímulos funcionales al logro de objetivos (Servera \& Llabrés, 2004), en oposición a los procesos atencionales en que la vigilancia depende de los atributos del estímulo como magnitud/intensidad, cantidad, ritmo de presentación y novedad (Cuervo \& Quijano, 2008). Las dificultades atencionales impiden al sujeto seleccionar estímulos cuya relevancia depende más de los objetivos socioculturales perseguidos, que de las características intrínsecas del estímulo. Cuervo \& Quijano (2008) evaluaron la velocidad de procesamiento de la información y la atención en niños con y sin TDAH, concluyendo que existen diferencias significativas en los tiempos de reacción en los grupos que presentan alguna alteración neuropsicológica, los cuales estarían relacionados con un déficit en la atención, que produce un enlentecimiento en la ejecución.

La inhibición también ha sido estudiada de manera preferente en el caso del TDAH. La inhibición parece ser una función ejecutiva que facilita la supresión o demora de respuestas automáticas en beneficio de conductas alternativas con mayor posibilidad de adaptación positiva al contexto (Aaron, Robbins \& Poldrack, 2004; Martín, Hernández, Rodríguez, García, Díaz \& Jiménez, 2012). Algunos autores han propuesto que el proceso de inhibición tiene dos manifestaciones, una de carácter conductual y otra de carácter cognitivo. La inhibición conductual se refiere a supresión, ralentización o corrección de pautas de comportamiento de escaso valor adaptativo (Aaron, Robbins \& Poldrack, 2004). La inhibición cognitiva supone un mecanismo por el cual el sistema ejecutivo bloquea el ingreso, a la memoria de trabajo, de un input sensorial no relativo a la tarea, favoreciendo de este modo el procesamiento de información relevante. Este segundo mecanismo ha sido propuesto como uno de los factores que explicaría el TDAH, vinculando el déficit de atención con una dificultad en la inhibición cognitiva de información irrelevante (De Beni, Palladino, Pazzaglia \& Cornoldi, 1998). Rubiales (2012) mostró, por medio de la evaluación de 60 niños entre 8 y 14 años de edad con y sin TDAH, que los niños con este trastorno demuestran dificultades superiores para inhibir las respuestas impulsivas que aquellos que no tienen TDAH.

Otra función ejecutiva mencionada por la literatura, como vinculada al TDAH es la flexibilidad cognitiva. Esta es referida a la capacidad para cambiar los criterios de selección o la habilidad para cambiar o alternar un conjunto de 
respuestas, aprender de los errores, utilizar estrategias alternativas, dividir la atención y procesar múltiples fuentes de información simultáneamente (Etchepareborda \& Mulas, 2004; Rodríguez et al., 2012). Rodríguez et al. (2012) sostiene que el rendimiento en la flexibilidad cognitiva presenta avances significativos entre los siete y quince años de edad, lo que da cuenta de un desarrollo más evidente en edades tempranas pero que se desacelera a partir de los 9 años y 3 meses, existiendo una relación lineal significativa entre la variable edad y la variable dependiente flexibilidad. La alteración de la flexibilidad cognitiva se relaciona estrechamente con trastornos en la discriminación atencional (estado de alerta y atención selectiva), el control de impulsos y el control de interferencia. Un ejemplo de ello, son los niños que padecen TDAH-HI, quienes presentan un déficit en la flexibilidad y la inhibición cognitiva y motora. Esta dificultad se encuentra caracterizada por la producción de un número significativamente superior de errores y de perseveraciones que los niños sin TDAH (Rubiales, 2012).

Si bien las escalas conductuales y los test neuropsicológicos para evaluar funciones ejecutivas son herramientas útiles para la detección del TDAH, diversos autores han señalado que existe un serio problema de sobrediagnóstico e infradiagnóstico en este trastorno (Carrasco, et al., 2012; Bernard, Servera \& Belmar, 2015; Sánchez-Trujillo \& Torres-López, 2018). Herranz (2006) muestra en un estudio que solo el $41 \%$ de los pediatras de Atención Primaria en España posee conocimientos suficientes para el diagnóstico adecuado del TDAH.

En Chile, el problema del sobrediagnóstico del TDAH ha recibido escasa atención, aunque algunos estudios han puesto de manifiesto que los criterios para diagnosticar el trastorno suelen no ser de carácter clínico y se sitúan más bien en las altas expectativas relativas al rendimiento escolar (FerrerUrbina, et al., 2017) o a una incorrecta observación de los síntomas declarados en el DSM-V, superando los parámetros poblacionales internacionales (Serón \& Rodríguez, 2017).

En el contexto chileno, el diagnóstico del TDAH no contempla la aplicación de test neuropsicológicos, y la observación conductual se hace con criterios diferentes a los señalados por el CIE-10 y el DSM-V. La normativa que rige la Educación Especial en Chile considera que el diagnóstico debe ser realizado por profesionales idóneos, sea este un neurólogo, psiquiatra, pediatra o un médico familiar, y un psicólogo, profesor de educación diferencial o psicopedagogo (Decreto №170/2010). 
El procedimiento sugerido por el Decreto 170/2010, indica que el diagnóstico de TDAH se realiza considerando, a) la clasificación y criterios de las revisiones más recientes del CIE-10 y complementariamente, del DSM-IV-R; b) un proceso de detección y derivación, que incluye anamnesis, observación directa del comportamiento, por al menos un semestre, dentro y fuera del aula por parte del profesor de asignatura o profesor de educación especial; evaluación pedagógica, entrevista a la familia, revisión de antecedentes escolares y evaluaciones previas de otros especialistas, elaboración de informe de derivación a un especialista, aplicación de pruebas en base a criterios como el Test de Conners; y c) un proceso de evaluación diagnóstica integral, que incluye un examen de salud y revisión de la historia médica del o la estudiante, una psicopedagógica que aporte información relevante del contexto escolar y familiar, determinación del grado de severidad, cumplimiento de criterios del CIE/DSM y un diagnóstico diferencial.

A la ausencia de evaluación neuropsicológica debe sumarse que, a pesar de la mención de los criterios diagnósticos para el TDAH contenidos en el CIE-10 y el DSM-V, la pauta de observación conductual sugerida es el Cuestionario de Conducta de Conners para Profesores, ampliamente difundida por internet y que no satisface los criterios diagnósticos recomendados internacionalmente.

El objetivo de este trabajo es estudiar las diferencias en la distribución de dos muestras de estudiantes con y $\sin$ TDAH, diagnosticadas con una escala de observación conductual a través de la evaluación de las funciones ejecutivas de atención, inhibición y flexibilidad cognitiva.

\section{MÉTODO}

\subsection{Diseño}

Esta es una investigación cuantitativa descriptiva no-experimental, cuyo objetivo es estudiar las diferencias entre estudiantes con y sin diagnóstico de TDAH mediante la aplicación de una batería neuropsicológica.

\subsection{Participantes}

Se conformó una muestra no probabilística por conveniencia de 132 estudiantes, 66 con diagnóstico clínico de TDAH y 66 controles, todos pertenecientes a un colegio particular-subvencionado de la Región de Biobío (Chile); el colegio atiende a cerca de 1.238 estudiantes. La edad de los alumnos de la muestra fluctúa entre 8 y 18 años; el promedio de edad fue $13,34(D E=2,46) ; 83,3 \%$ de los estudiantes son mujeres y $16,7 \%$ son 
hombres. Todos los estudiantes cursaban entre 3ํaño de Enseñanza Básica y IIIo de Enseñanza Media. Como criterios de inclusión se consideró, (1) a estudiantes con asistencia regular al establecimiento, (2) tener diagnóstico de TDAH avalado por educadora diferencial y neurólogo o psiquiatra. Como criterios de exclusión se consideró que los estudiantes (1) no presentasen trastorno del espectro autista, (2) funcionamiento intelectual limítrofe, (3) discapacidad intelectual o (4) trastorno específico del lenguaje. La información diagnóstica suele ser altamente confidencial, cuestión por la que solo hubo acceso a una lista de estudiantes mencionando la presencia de trastornos y no a las pruebas utilizadas para su diagnóstico ni a las puntuaciones obtenidas. La revisión del listado permitió identificar a 133 estudiantes con diagnóstico de TDAH; este se realiza a través de la cumplimentación del Cuestionario de Conducta Conners para Profesores, en su versión abreviada y de libre acceso en internet; el cuestionario es completado por un profesor y enviado a un neurólogo o pediatra, el que realiza una evaluación clínica del estudiante. No consta la utilización de test neuropsicológicos en el proceso. Finalmente, la información diagnóstica debe ser informada al Ministerio de Educación. La prevalencia de TDAH en el establecimiento es de 10,74 \%.

Se envió una comunicación escrita a los padres/apoderados de los 133 estudiantes con diagnóstico de TDAH para que firmaran el consentimiento de participación. Fueron recibidos 81 respuestas favorables. 15 estudiantes no rindieron todas las pruebas contempladas, cuestión por la que fueron excluidos de la muestra final, que quedó conformada por $\mathrm{N}=66$, de los cuales un $18,2 \%$ corresponden a hombres y el $81,8 \%$ a mujeres, con un promedio de edad de 13,5 años y una desviación estándar de 2,7.

Paralelamente, se conformó un grupo control con estudiantes que cursasen entre $3^{\circ}$ año de Enseñanza Básica y $1 I^{\circ}$ año de Enseñanza Media, con criterios de exclusión idénticos al del grupo de estudio. El procedimiento de selección se realizó con la intención de configurar un grupo de estudiantes sin TDAH de tamaño equivalente al grupo en estudio, por tanto, si un curso tenía tres niños y una niña con TDAH, se buscaba, de forma aleatoria, en el mismo curso a tres niños y una niña que no tuvieran TDAH y que cumplieran con los criterios de exclusión. A todos los padres/apoderados del grupo control se les envió una comunicación escrita para que firmaran el consentimiento de participación. Como en el caso anterior, algunos padres/apoderados no firmaron el consentimiento de participación y algunos niños no pudieron rendir todas las pruebas, cuestión que se resolvió reemplazando a los sujetos por otros de características equivalentes. El grupo control quedó conformado 
por un $\mathrm{N}=66$, de los cuales $14 \%$ son hombres y $86 \%$ son mujeres, con un promedio de edad de 13,5 años y desviación estándar de 2,3. De esta manera, la muestra definitiva quedó compuesta por 132 estudiantes de 8 a 18 años, con un promedio de edad de 13,5 años, $84 \%$ mujeres y $16 \%$ hombres. La disparidad en la cantidad de mujeres y hombres se debe a que el establecimiento ha atendido históricamente a niñas, solo en los últimos años se han incorporado niños a la matrícula escolar, específicamente a los cursos iniciales, esto es de pre-escolar (4 a 5 años) a 70 Básico (12 años).

Todos los padres/madres/tutores de los estudiantes incluidos en este estudio, aceptaron la participación de sus hijos/hijas/pupilos a través de la firma de un consentimiento informado, en que declaraban comprender la naturaleza y objetivos de la investigación y los derechos que les asisten en estos casos. Los estudiantes participantes otorgaron su asentimiento informado de participación. En todo el proceso evaluativo se respetaron las normas éticas de investigación con personas contenidas en la Declaración de Helsinki y del Comité de Ética de la Universidad Católica de la Santísima Concepción.

\subsection{Instrumentos}

\subsubsection{Test de Cinco Dígitos (FDT)}

Es un test neuropsicológico que se utiliza para medir inhibición y flexibilidad cognitiva (Sedó, 2004, 2007). El test presenta cuatro tareas visuales; cada tarea se compone de 50 ítems contenidos en pequeños recuadros organizados en una matriz de $5 \times 10$. Las dos primeras tareas miden procesos automáticos como lectura de dígitos y conteo de asteriscos; las otras dos tareas miden procesos ejecutivos de elección de estímulos para el procesamiento y alternancia de estímulos para el procesamiento. Este test ha sido ampliamente recomendado en lugar de otros semejantes, dado sus características de aplicación y corrección. Algunos autores (Rodríguez, Jiménez, Díaz, García, Martín \& Hernández, 2012) han detectado que las personas con un inadecuado nivel de decodificación lectora, analfabetos o hablantes de una lengua diferente al castellano suelen obtener puntuaciones desajustadas en pruebas semejantes tipo Stroop, cuestión por la cual han preferido el FDT. El coeficiente de fiabilidad del instrumento fue calculado a partir del procedimiento de dos mitades; el valor resultante fue corregido con la fórmula de Spearman-Brown. En base a este procedimiento se obtuvieron coeficientes de .95 en lectura, .94 en conteo, .93 en elección y .92 en alternancia. 


\subsubsection{Test de Percepción de Diferencias Caras-R}

Es un test neuropsicológico que se utiliza para la evaluación de la atención. Consta de 60 elementos gráficos formados por tres estímulos; cada estímulo gráfico está formado por tres dibujos de caras con rasgos diferenciadores de boca, cejas y pelo. Dos de las caras son iguales y una es diferente; el objetivo de la tarea es marcar la figura que es diferente a las otras dos (Thurstone \& Yela, 2012). En la versión utilizada para este estudio, se considera el número de aciertos y los errores cometidos por el sujeto evaluado, pudiendo obtener así la cantidad de aciertos netos y un índice de control de impulsividad, lo cual permite relacionar el rendimiento obtenido con el estilo de respuesta (Thurstone \& Yela, 2012). Cada una de las puntuaciones entregadas se puede traducir a percentiles y eneatipos de acuerdo a lo establecido en el manual de aplicación. La fiabilidad de la prueba se realizó utilizando el coeficiente alpha de Cronbach cuyo valor obtenido en la muestra global fue de 0,91.

\subsection{Procedimiento}

Se realizó una entrevista y petición formal a los directivos de un colegio particular de la ciudad de Concepción, en Chile, que recibe subvención estatal, para solicitar la autorización de realizar la investigación. El estudio se llevó a cabo durante el mes de noviembre y diciembre 2018. Se inició con una revisión de las fichas y carpetas de aquellos alumnos que pertenecen al Programa de Integración Escolar del establecimiento. Se identificó al grupo de estudiantes que está diagnosticado con TDAH. Luego, se conformó un grupo control equivalente, de tal manera que existieran la misma cantidad de niñas y niños, con y sin TDAH. Si un curso tenía tres niñas con el diagnóstico, se eligieron al alzar, tres niñas que no tuvieran TDAH en el mismo curso. Se procedió a enviar consentimientos informados a los padres para que los estudiantes pudiesen participar del proyecto. Tras la recepción de estos, se conformó una muestra de 66 alumnos con TDAH de $3^{\circ}$ básico a $111^{\circ}$ medio y 71 estudiantes sin TDAH considerando los mismos niveles académicos. Una vez conformados los grupos se aplicó a cada estudiante una batería neuropsicológica, a ambos grupos para evaluar atención, inhibición y flexibilidad cognitiva. El Test de Percepción de Diferencias Caras-R se aplicó de manera colectiva en grupos de hasta 8 estudiantes. El Test de los Cinco Dígitos se aplicó de manera individual. El criterio de corte se estableció en el percentil 15. El criterio de corte para el diagnóstico se ajustó al orden siguiente: a) estudiantes con rendimiento $\leq 15$ percentil en atención y rendimiento > 15 percentil en inhibición eran categorizados como TDAH-l; b) estudiantes con rendimiento $>15$ percentil en atención y rendimiento $\leq 15$ percentil en 
inhibición eran categorizados como TDAH-HI; c) estudiantes con rendimiento 15 percentil en atención y rendimiento $\leq 15$ percentil en inhibición eran categorizados como TDAH-C; d) estudiantes con rendimiento $>15$ percentil en atención y rendimiento $>15$ percentil en inhibición eran categorizados como SIN-TDAH. Si bien el punto de corte suele fijarse en $p<30$, se decidió utilizar un criterio más estricto para evitar falsos positivos dada la condición sociocultural de los estudiantes evaluados.

El análisis de los datos se realizó con programa Statistical Package for the Social Sciences (SPSS) Statistics 23.

\subsection{Análisis estadísticos}

Para analizar las características de los grupos con y sin diagnóstico de TDAH, se calcularon estadísticos descriptivos, para ambos grupos por separado. La determinación de la normalidad en la distribución de los datos en las tres variables estudiadas (atención, inhibición y flexibilidad cognitiva) se realizó con la prueba de normalidad de Kolmogorov-Smirnov. Las diferencias entre las variables categóricas de diagnóstico (con y $\sin$ TDAH) y subtipo se analizaron usando la prueba de Chi-cuadrado $\left(\mathrm{X}^{2}\right)$. Como los datos no se distribuyeron normalmente, la prueba $\mathrm{U}$ de Mann-Whitney se usó para explorar las diferencias en la distribución de la mediana entre los grupos con y sin diagnóstico de TDAH (Conners), y los grupos subtipo TDAH. En cada análisis el valor $\mathrm{p}<0.05$ se consideró estadísticamente significativo. El análisis estadístico se realizó con Statistical Package for Social Sciences (SPSS) versión 23.

\section{RESULTADOS}

El procesamiento de los datos contempló el análisis estadístico descriptivo de las variables atención, inhibición y flexibilidad cognitiva, en función a la pertenencia a los grupos con y sin diagnóstico de TDAH. Los resultados mostraron que, para el grupo TDAH, las variables de atención $(M=37,62)$, inhibición $(M=35,48)$ y flexibilidad cognitiva $(M=30,09)$, muestran un $p>30$ en las medias baremado en percentiles, medida habitualmente aceptada para diferencias clínicamente significativas. Basados en estos datos, se pudo observar que, tanto en el grupo con TDAH como en el grupo sin TDAH, había sujetos que, por su rendimiento en atención e inhibición, no pertenecían al grupo de origen. Los análisis descriptivos se exponen en la Tabla 1. 
Tabla 1.

Descriptivos

\begin{tabular}{|c|c|c|c|c|c|c|c|}
\hline & & \multirow[t]{2}{*}{$\mathbf{N}$} & \multirow[t]{2}{*}{ MEDIA } & \multirow{2}{*}{$\begin{array}{l}\text { DESVIACIÓN } \\
\text { TÍPICA }\end{array}$} & \multirow{2}{*}{$\begin{array}{l}\text { ERROR } \\
\text { TíPICO }\end{array}$} & \multicolumn{2}{|c|}{$\begin{array}{l}\text { INTERVALO DE CONFIANZA PARA LA } \\
\text { MEDIA AL 95\% }\end{array}$} \\
\hline & & & & & & LÍMITE INFERIOR & LÍMITE SUPERIOR \\
\hline \multirow{3}{*}{ ATE_PC } & TDAH & 66 & 37,62 & 28,94 & 3,56 & 30,50 & 44,73 \\
\hline & NO_TDAH & 66 & 48,65 & 30,28 & 3,72 & 41,20 & 56,09 \\
\hline & Total & 132 & 43,13 & 30,02 & 2,61 & 37,96 & 48,30 \\
\hline \multirow{3}{*}{ INH_PC } & TDAH & 66 & 35,48 & 32,56 & 4,00 & 27,47 & 43,49 \\
\hline & NO_TDAH & 66 & 52,84 & 30,87 & 3,80 & 45,25 & 60,43 \\
\hline & Total & 132 & 44,16 & 32,78 & 2,85 & 38,52 & 49,81 \\
\hline \multirow{3}{*}{ FLEX_PC } & TDAH & 66 & 30,09 & 28,98 & 3,56 & 22,96 & 37,21 \\
\hline & NO_TDAH & 66 & 47,60 & 31,50 & 3,87 & 39,86 & 55,35 \\
\hline & Total & 132 & 38,84 & 31,41 & 2,73 & 33,44 & 44,25 \\
\hline
\end{tabular}

La evaluación neuropsicológica permitió re categorizar a los participantes $(n=132)$ de acuerdo al subtipo de TDAH $(p<15)$. Se utilizó una tabla de contingencia de $2 \times 2$ para agrupar los datos de los grupos con y sin TDAH, basados en el diagnóstico realizado con el Cuestionario de Conducta Conners para Profesores y los resultados de la evaluación de funciones ejecutivas que dio lugar a la categorización por subtipos. Los datos pueden observarse en la Tabla 2.

Tabla 2

Tabla de contingencia DIAG_CONNERS * SUBTIPO

\begin{tabular}{cccccccc}
\hline & & \multicolumn{5}{c}{ SUBTIPO } \\
\cline { 3 - 7 } & & & TDAH_I & TDAH_HI & TDAH_C & NO_TDAH & TOTAL \\
\hline \multirow{3}{*}{ DIAG_CONNERS } & \multirow{3}{*}{ TDAH } & Recuento & 12 & 16 & 9 & 29 & 66 \\
& & Frecuencia esperada & 14,0 & 13,0 & 5,5 & 33,5 & 66,0 \\
& \multirow{2}{*}{ NO_TDAH } & Recuento & 16 & 10 & 2 & 38 & 66 \\
& & Frecuencia esperada & 14,0 & 13,0 & 5,5 & 33,5 & 66,0 \\
\cline { 3 - 7 } & \multirow{2}{*}{ Total } & Recuento & 28 & 26 & 11 & 67 & 132 \\
& & Frecuencia esperada & 28,0 & 26,0 & 11,0 & 67,0 & 132,0 \\
\hline
\end{tabular}

En base a la tabla de contingencia, se calculó $X^{2}$ para determinar si el valor observado de la variable diagnóstico depende del valor observado de la variable subtipo. Los resultados en el análisis de $X^{2}$ (sig 0.055, $\left.p>, 05\right)$, con un $\mathrm{H}_{1}$ de relación entre diagnóstico y subtipo, no permiten rechazar $\mathrm{H}_{0}$. Los resultados quedan recogidos en la Tabla 3. Esta observación es congruente con los datos porcentuales que pueden extraerse de la Tabla 2 y que muestran un $43,93 \%$ de los estudiantes no perteneciente al grupo con diagnóstico TDAH informado por el colegio. De modo semejante, el porcentaje de estudiantes no pertenecientes al grupo sin diagnóstico TDAH informado por el colegio, es de $42,42 \%$. 
Tabla 3

Pruebas de Chi-cuadrado

\begin{tabular}{cccc}
\hline & VALOR & GL & $\begin{array}{c}\text { SIG. ASINTÓTICA } \\
\text { (BILATERAL) }\end{array}$ \\
\hline Chi-cuadrado de Pearson & $7,620 \mathrm{a}$ & 3 &, 055 \\
Razón de verosimilitudes & 8,001 & 3 &, 046 \\
Asociación lineal por lineal &, 239 & 1 &, 625 \\
$\mathrm{~N}$ de casos válidos & 132 & & \\
\hline
\end{tabular}

a. 0 casillas $(0,0 \%)$ tienen una frecuencia esperada inferior a 5. La frecuencia mínima esperada es 5,50.

Finalmente, ante la falta de distribución normal de la muestra, se lleva a cabo la prueba no-paramétrica de diferencia de medianas U Mann-Whitney. Se utiliza este estadígrafo para verificar el modo que cada variable en estudio se relaciona con el subtipo inatento, hiperactivo/impulsivo y combinado, y con el grupo sin TDAH. Para la Prueba de U Mann-Whitney se utilizó un nivel de significancia del $95 \%$.

Los resultados muestran diferencias significativas entre los subtipos dependiendo de la variable en estudio. La atención parece estar más presenta en los subtipos inatento y combinado; por su parte la inhibición parece más presente en los subtipos hiperactivo/impulsivo y combinado. La flexibilidad cognitiva no parece seguir un patrón identificable de distribución. El resumen de los datos se presenta en la Tabla 4.

Tabla 4

Estadísticos de contraste

\begin{tabular}{cccc}
\hline TDAH_I / TDAH_HI & ATE_PC & INH_PC & FLEX_PC \\
\hline U de Mann-Whitney &, 000 &, 000 & 237,500 \\
W de Wilcoxon & 406,000 & 351,000 & 588,500 \\
Z & $-6,340$ & $-6,331$ & $-2,201$ \\
Sig. asintót. (bilateral) &, 000 &, 000 &, 028 \\
\hline TDAH_I/ TDAH_C & ATE_PC & INH_PC & FLEX_PC \\
\hline U de Mann-Whitney & 151,000 &, 000 & 64,500 \\
W de Wilcoxon & 217,000 & 66,000 & 130,500 \\
Z &,- 097 & $-4,820$ & $-2,813$ \\
Sig. asintót. (bilateral) &, 923 &, 000 &, 005 \\
\hline TDAH_I / NO_TDAH & ATE_PC & INH_PC & FLEX_PC \\
\hline U de Mann-Whitney & 11,500 & 773,000 & 687,500 \\
W de Wilcoxon & 417,500 & 1179,000 & 1093,500 \\
Z & $-7,580$ & $-1,351$ & $-2,050$ \\
Sig. asintót. (bilateral) &, 000 &, 177 &, 040 \\
\hline TDAH_HI / TDAH_C & ATE_PC & INH_PC & FLEX_PC \\
\hline
\end{tabular}




\begin{tabular}{cccc}
\hline TDAH_I / TDAH_HI & ATE_PC & INH_PC & FLEX_PC \\
\hline U de Mann-Whitney &, 000 & 119,000 & 133,500 \\
W de Wilcoxon & 66,000 & 185,000 & 199,500 \\
Z & $-4,770$ &,- 814 &,- 319 \\
Sig. asintót. (bilateral) &, 000 &, 416 &, 749 \\
\hline TDAH_HI / NO_TDAH & ATE_PC & INH_PC & FLEX_PC \\
\hline U de Mann-Whitney & 488,500 & 2,000 & 400,000 \\
W de Wilcoxon & 839,500 & 353,000 & 751,000 \\
Z & $-3,283$ & $-7,452$ & $-4,040$ \\
Sig. asintót. (bilateral) &, 001 &, 000 &, 000 \\
\hline TDAH_C / NO_TDAH & ATE_PC & INH_PC & FLEX_PC \\
\hline U de Mann-Whitney & 5,000 & 1,500 & 66,500 \\
W de Wilcoxon & 71,000 & 67,500 & 132,500 \\
Z & $-5,230$ & $-5,278$ & $-4,346$ \\
Sig. asintót. (bilateral) &, 000 &, 000 &, 000 \\
\hline & & & \\
\hline
\end{tabular}

\section{DISCUSIÓN}

Las medias de atención e inhibición están sobre las dos desviaciones estándar ( $M=37,62 ; M=35,48$; respectivamente), criterio usualmente utilizado para el diagnóstico de TDAH; lo mismo ocurre con la flexibilidad cognitiva ( $M=30,09)$. Esto parece sugerir que en el grupo TDAH_CONNERS habría algunos sujetos que no cumplen con los criterios diagnósticos sugeridos para la evaluación de TDAH.

La evaluación neuropsicológica y la evaluación con el Cuestionario de Conducta Conners mostraron discrepancias; la coincidencia en el diagnóstico de TDAH con ambos procedimientos es de 56,06 \%; la coincidencia diagnóstica en el grupo sin TDAH es de 57,57\%. Esto plantea la posibilidad de que ambos grupos presenten casos de sobre e infradiagnóstico que alcanzan el $43,93 \%$ y el $42,42 \%$, respectivamente. Estos datos parecen plantear que las variables categóricas entregadas por ambos procedimientos diagnósticos son independientes. Frente a esta situación, el análisis de diferencias entre las frecuencias observadas y esperadas mostró que efectivamente ambas categorías nominales son independientes. A pesar de los datos que mostrarían baja coincidencia entre los tipos de diagnóstico, la reagrupación de los casos según la evaluación neuropsicológica mostró que la distribución original de la muestra (TDAH, $n=66$; NO_TDAH, $n=66$ ) prácticamente no varió con una distribución de TDAH $(n=65)$ y NO_TDAH $(n=67)$. La mayor proporción de mujeres en la muestra impide contrastar los datos encontrados con la prevalencia aceptada de TDAH y la distribución de sus subtipos; en este caso encontramos que un 43,07 \% de los estudiantes evaluados en atención e 
inhibición correspondieron al subtipo con predominio inatento, un 40,00\% al subtipo hiperactivo/impulsivo y un $16,96 \%$ al subtipo combinado.

El análisis de comparación de medianas muestra que existen diferencias significativas entre los subtipos de TDAH de acuerdo a las variables nucleares del trastorno (atención e inhibición). El presente estudio detectó que las dificultades clínicamente significativas en atención e inhibición se presentan específicamente en los subtipos inatento y combinado, y en el subtipo hiperactivo/impulsivo y combinado, respectivamente. En otras palabras, ambas variables son especialmente útiles para (1) diagnosticar TDAH (Barkley, 1997a, 1997b; Sabagh-Sabbagh, 2008; Martín et al., 2012; Rebollo \& Montiel, 2006; Rubiales, 2012), y (2) determinar el subtipo. El Cuestionario Conners utilizado por el colegio para diagnosticar TDAH no permite determinar subtipo y sus resultados presentan discrepancias con los obtenidos por la evaluación neuropsicológica.

La relación entre la flexibilidad cognitiva y el diagnóstico de TDAH por subtipos es más confusa. Los resultados de la comparación de medianas muestra que existen diferencias significativas cada vez que se contrastan la categoría TDAH (en cualquiera de sus subtipos) y la categoría NO_TDAH. Esto puede indicar que la flexibilidad cognitiva podría ser funcional para detectar TDAH de manera general (Etchepareborda y Mulas, 2004; Etchepareborda et al., 2004: Rubiales, 2012 y Rodríguez et al., 2012), pero no es clara su relación con los subtipos como para orientar su identificación.

Tal como lo confirman las investigaciones previas (Mas-Pérez, 2009; Peláez, 2009; García \& Domínguez, 2012; Ripol-Baixas, 2015), actualmente existe en una situación preocupante de sobrediagnóstico del TDAH. Los autores sostienen que es habitual la confusión entre el TDAH y otras entidades, lo cual puede deberse a la falta de una metodología clínica para el diagnóstico, ya que solo se considera el historial clínico o el comportamiento del niño analizado en base a escalas de observación conductual como el Cuestionario de Conducta Conners (Valdivieso, Cornejo \& Sánchez, 2000). García y Domínguez (2012), señalan como una de las posibles causas del sobrediagnóstico e infradiagnóstico, la existencia de instrumentos poco eficaces. Ferrer-Urbina, et al., (2017) han hecho notar que la lista de síntomas conductuales para el diagnóstico del TDAH es extensa y redundante, cuestión que dificulta la detección, en especial si esta es realizada por profesionales con escasa experiencia en el área. Es probable que las altas expectativas de rendimiento escolar, que padres y profesores depositan en los niños, aumenten la necesidad de encontrar explicaciones clínicas al fracaso escolar. 
Otro problema es el que plantea el infradiagnóstico detectado. Si bien, el infradiagnóstico ha sido mencionado por algunos autores (Bernard, Servera \& Belmar, 2015), no ha sido señalado como un problema de la misma magnitud que el sobrediagnóstico. En este caso, la evidencia de estudiantes con perfil cognitivo de TDAH, y que pasaron desapercibidos al equipo psicoeducativo del colegio, no fue previsto por este estudio y menos en el porcentaje detectado. Esta situación parece referir al mecanismo con que los profesores derivan a sus estudiantes al proceso diagnóstico. Para este caso, el 24,24 \%, de los estudiantes infradiagnosticados presentaron un perfil cognitivo de TDAH-I, es decir, estudiantes con dificultades en el control atencional pero que no generan problemas conductuales en la clase; el otro grupo (TDAH-HI y TDAH-C), que representa al $18,18 \%$ de los estudiantes infradiagnosticados, presenta una situación análoga. Si aceptamos que la inhibición se compone de una inhibición conductual (relativa a la hiperactividad/impulsividad) y de una inhibición cognitiva (relativa al procesamiento de la información), debemos aceptar que el test seleccionado para medir inhibición (FDT) se orienta sobre la inhibición cognitiva. Barkley (1997b), sostiene que el problema central del TDAH es la dificultad de las personas para impedir que la información irrelevante ingrese al sistema cognitivo durante el procesamiento. No existe evidencia sólida que vincule la inhibición conductual con la inhibición cognitiva. Si bien, el 18,18 \% de los estudiantes infradiagnosticados presenta algún grado de dificultad en la inhibición, esto no significa que presenten desinhibición conductual.

Este conjunto de observaciones, refuerza lo sostenido por otros autores (Etchepareborda, Paiva-Barón \& Abad, 2009; Tirapu-Ustárroz, García-Molina, Luna-Lario \& Periáñez, 2012; López-Soler \& Romero-Medina, 2013) respecto al valor de la evaluación neuropsicológica de las funciones ejecutivas en el TDAH. No obstante, la utilización de escalas de observación del comportamiento actualizadas, como la Vanderbilt ADHD Rating Scale debiera incluirse en un proceso de evaluación integral.

Parece necesario entonces, mejorar el estándar evaluativo para detección del TDAH, incorporando escalas de observación conductual alineadas con la investigación actual y una batería neuropsicológica sólida. Tal como plantea Carreño-Álvarez \& Gatica-Ferrero (2019), las escalas de observación conductual y la evaluación neuropsicológica no son elementos contrapuestos, sino que forman parte de un continuo que aporta solidez al diagnóstico del TDAH.

Conflictos de intereses: Los autores declaran no tener conflictos de interés en ninguna de las etapas del presente estudio. 
Agradecimientos: PROYECTO INDINO4/2018. Dirección de Investigación, Universidad Católica de la Santísima Concepción. Programa de Magíster en Psicopedagogía y Educación Especial, Universidad Católica de la Santísima Concepción. CONICYT FCHA/21190754-2019

Financiamiento: Estudio financiado por el Proyecto INDIN04/2018 de la Dirección de Investigación de la Universidad Católica de la Santísima Concepción, Chile.

\section{REFERENCIAS}

Aaron, A., Robbins, T. \& Poldrack, R. (2004). Inhibition and the right inferior frontal cortex. Trends in cognitive sciences, 8(4), 170-177. http://dx.doi.org/10.1016/j. tics.2004.02.010

Abad-Mas, L., Caloca-Català, O., Mulas, F., \& Ruiz-Andrés, R. (2017). Comparación entre el diagnóstico del trastorno por déficit de atención/hiperactividad con el DSM-5 y la valoración neuropsicológica de las funciones ejecutivas. Revista de neurología, 64(1), 95-100. https://dialnet.unirioja.es/servlet/articulo?codigo=5986468

Asociación Americana de Psiquiatría (APA). (2014). Manual diagnóstico y estadístico de los trastornos mentales. 5o Edición. España: Editorial Médica Panamericana. https://www.medicapanamericana.com/Libros/Libro/4949/DSM5-Manual-Diagnostico-y-Estadistico-de-los-Trastornos-Mentales.html

Barkley R. A. (1997a). Behavioural Inhibition, Sustained Attention, and Executive Functions: Constructing a unifying theory of AD/HD. Psychological Bulletin, 121(1), 65-94. http://dx.doi.org/10.1037/0033-2909.121.1.65

Barkley, R. A. (1997b). ADHD and the nature of self-control. New York, US: Guilford Press.

Barkley, R. A. (2009). Avances en el diagnóstico y la subclasificación del Trastorno por Déficit de Atención/Hiperactividad: Qué puede pasar en el futuro respecto al DSM-V. Rev Neurol, 48(Supl2), 101-106. https://doi.org/10.33588/rn.48S02.2009003

Becker, S. P. (2013). Topical review: Sluggish cognitive tempo: research findings and relevance for pediatric psychology. Journal of Pediatric Psychology, 38(10), 1051-1057. https://doi.org/10.1093/jpepsy/jst058

Bernard, M. M., Servera, M. \& Belmar, M. (2015). La dimensión Sluggish Cognitive Tempo: el estado de la cuestión. Revista de Psicología Clínica con Niños y Adolescentes, 2(2), 95-106. https://www.revistapcna.com/sites/default/files/01-1_ bernad_sct.pdf

Cardo, E., Servera, M., Vidal, C., de Azua B, Redondo, M. \& Riutort, L. (2011). The influence of different diagnostic criteria and the culture on the prevalence of attention deficit hyperactivity disorder [in Spanish]. Rev Neurol.; 52:S109-17. https://www.ncbi.nlm.nih.gov/pubmed/21365591

Carrasco, X., Daiber, F., Pothhamer, P., Huertu, D., Andrade, C., Apazo P., Paz, L., Pothhamer, F. y Abaitiz, F. (2012). Trastorno por déficit de atención e hiperactividad en niños aymara: primera aproximación clínica. Rev Med Chile; 140, 1409-1416. http://dx.doi.org/10.4067/S0034-98872012001100005

Carreño-Álvarez, M. \& Gatica-Ferrero, S. (2019). Determinación de la correlación en el uso del Cuestionario de Vanderbilt y la aplicación de pruebas neuropsicológicas para el diagnóstico del Trastorno por Déficit Atencional. Revista de Salud Pública y Nutrición, 18(2), 1-7. https://doi.org/10.29105/respyn18.2-1

Cuervo, M. \& Quijano, M. (2008). Las alteraciones de la atención y su rehabilitación en trauma craneoencefálico. Revista Pensamiento Psicológico, 4(11), 167-182. https:// www.redalyc.org/articulo.oa?id=801/80111671011 
De Beni, R., Palladino, P., Pazzaglia, F. \& Cornoldi, C. (1998). Increases in intrusion errors and working memory deficit of poor comprehenders. The Quarterly Journal of Experimental Psychology 51A (2), 305-320. https://doi.org/10.1080/713755761

De la Barra, F., Vicente, B., Saldivia, S. \& Melipillán, R. (2013). Epidemiology of ADHD in Chilean children and adolescents. ADHD Atten Def Hyp Disord; 5, 1-8. htpps://doi. org/10.1007/s12402-012-0090-6.

Decreto 170/2009 Diario Oficial de la República de Chile, Santiago de Chile, 21 de abril de 2010. https://www.leychile.cl/N?i=1012570\&f=2010-08-25\&p=

Etchepareborda, M. C. \& Mulas, F. (2004). Flexibilidad cognitiva, síntoma adicional del trastorno por déficit de atención con hiper-actividad. ¿Elemento predictor terapéutico? Rev Neurol, 38(1), 97-102. https://doi.org/10.33588/rn.38S1.2004041

Etchepareborda, M. C., Mulas, F., Capilla-González, A, Fernández-González, S., Campo, P., Maestú, F., Fernández-Lucas, A. \& Ortiz, T. (2004). Sustrato neurofuncional de la rigidez cognitiva preliminares. Rev Neurol, 38(1), 145-148. https://doi. org/10.33588/rn.38S1.2004080

Etchepareborda, M. C., Paiva-Barón, H. \& Abad L. (2009). Ventajas de las baterías de exploración neuropsicológica en el trastorno por déficit de atención/hiperactividad. Rev Neurol; 48, 89-93. https://doi.org/10.33588/rn.48502.2009073

Ferrer-Urbina, R., Chavéz, K. A., Gallardo, C., Loredo, G. y Meneses, K. (2017). Apreciación diagnóstica de profesores del Trastorno de Déficit Atencional con Hiperactividad (TDAH) en escolares de Enseñanza Básica pertenecientes a establecimientos municipalizados de la ciudad de Arica. Salud \& Sociedad, 8(1), 52-65. https://doi.org/10.22199/S07187475.2017.0001.00004

Flores-Aréchiga, A., Garza-González, M., LLaca-Díaz, J., \& Gómez Espinel, I. (2016). Comparación de cuatro instrumentos utilizados en la evaluación de los trastornos por déficit de atención e hiperactividad. Revista de Salud Pública y Nutrición, 15(2), 7. file:///C:/Users/profe-x4/Downloads/9-17-1-SM\%20(1).pdf

García, J. \& Domínguez, J. (2012). ¿Existe un sobrediagnóstico del trastorno de déficit de atención e hiperactividad (TDAH)? Evid Pediatr, 8(51), 1-5. http://www.evidenciasenpediatria.es/EnlaceArticulo?ref=2012;8:51

García, T., González-Castro, P., Rodríguez, C., Cueli, M., Álvarez, D. \& Álvarez, L. (2014). Alteraciones del funcionamiento ejecutivo en el trastorno por déficit de atención con hiperactividad y sus subtipos. Revista Psicología Educativa, 20(1), 23-32. https://doi.org/10.1016/j.pse.2014.05.003

García-Noriega, A. \& Bárcena, C. (2013). Valoración categorial del trastorno por Déficit de atención /hiperactividad, mediante la Escala de evaluación Vanderbilt en padres. Revista Enfermería CyL, 5(1), 32-41. http://www.revistaenfermeriacyl.com/index. $\mathrm{php} /$ revistaenfermeriacyl/article/view/71/78

Herranz, B. (2006). Trastorno por déficit de atención e hiperactividad: conocimientos y forma de proceder de los pediatras de Atención Primaria. Rev Pediatr Aten Primaria, 8(4), 217-239. http://archivos.pap.es/files/1116-600-pdf/625.pdf

Langberg, J. M., Vaughn, A. J., Brinkman, W. B., Froehlich, T., \& Epstein, J.N. (2010). Clinical utility of the Vanderbilt ADHD Rating Scale for ruling out comorbid learning disorders. Pediatrics, 126(5), e1033-e1038. https://doi.org/10.1542/peds.20101267

López I., Rodillo E. \& Kleinsteuber K. (2008). Neurobiología y diagnóstico del Trastorno por Déficit de Atención. Rev. Med. Clin. Condes, 19, 511-524. http://www.clc. cl/Dev_CLC/media/Imagenes/PDF\%20revista\%20m\%C3\%A9dica/2008/5\%20 nOV/06NEUROBIOLOGIA-6.pdf 
López-Soler, C. \& Romero-Medina, A., coords. (2013). TDAH y trastornos del comportamiento en la infancia y la adolescencia. Clínica, diagnóstico, evaluación y tratamiento. Madrid: Pirámide.

Martín, R., Hernández, S., Rodríguez, C., García, E., Díaz, A. \& Jiménez, J. (2012). Datos normativos para el Test de Stroop: patrón de desarrollo de la inhibición y formas alternativas para su evaluación. European Journal of Education and Psychology, 5(1), 39-51. https://www.redalyc.org/articulo.oa?id=1293/129324775004

Mas-Pérez, C. (2009). El TDAH en la Práctica Clínica Psicológica. Clínica y Salud, 20(3), 249-259. https://www.redalyc.org/articulo.oa?id=1806/180613881006

McCarthy, S., Wilton, L., Murray, M.L., Hodgkins, P., Asherson, P. \& Wong, I.C. (2012). The epidemiology of pharmacologically treated attention déficit hyperactivity disorder (ADHD) in children, adolescents and adults in UK primary care. BMC Pediatr, 12:78. Medline: 22712630. https://doi.org/10.1186/1471-2431-12-78

McKeown, R.E., Holbrook, J.R., Danielson, M.L., Cuffe, S.P., Wolraich, M.L., \& Visser, S.N. (2015). The Impact of Case Definition on Attention-Deficit/Hyperactivity Disorder Prevalence Estimates in Community-Based Samples of School-Aged Children. Journal of the American Academy of Child \& Adolescent Psychiatry, 54(1), 53-61. https://doi.org/10.1016/j.jaac.2014.10.014

Mulas, F., Etchepareborda, M. C., Abad-Mas, L., Díaz, A., Hernández, S., Pascuale, A., \& Ruiz, A., (2006). Trastornos neuropsicológicos de los adolescentes afectos de trastorno por déficit de atención e hiperactividad. Rev Neurol, 43(1), 71-81. https:// doi.org/10.33588/rn.43S01.2006388

Nigg, J. (2001). Is ADHD a Dishinibitory Disorder? Psychological Bulletin, 127, 571-598. https://doi.org/10.1037/0033-2909.127.5.571

Peláez, G. P. (2009). Hiperactividad: ¿Síntoma o trastorno? ¿De qué o de quién? Revista Electrónica Psiconex, 1(2), 1-17. http://hdl.handle.net/10495/2814

Rebollo, M. A. \& Montiel, S. (2006). Atención y funciones ejecutivas. Rev Neurol, 42(2), 3-7. https://doi.org/10.33588/rn.42S02.2005786

Ripol-Baixas, B. (2015). Detección e intervención del TDAH en la Educación [Tesis de pregrado]. Universidad Internacional de la Rioja, Barcelona. https://reunir.unir.net/ handle/123456789/2852

Rodríguez, C., Jiménez, J., Díaz, A., García, E., Martín, R. \& Hernández, S. (2012). Datos normativos para el Test de los Cinco Dígitos: desarrollo evolutivo de la flexibilidad en Educación Primaria. European Journal of Education and Psychology, 5(1), 27-38. https://www.redalyc.org/articulo.oa?id=1293/129324775003

Rubiales, J. (2012). Análisis de la flexibilidad cognitiva y la inhibición en niños con TDAH [Tesis doctoral]. Universidad Nacional de Mar del Plata, Buenos Aires. http://www. mdp.edu.ar/psicologia/psico/doctorado/tesis/rubiales.pdf

Sabagh-Sabbagh, S. (2008). Solución de problemas aritméticos redactados y control inhibitorio cognitivo. Univ. Psychol., 7(1), 215-28. https://www.redalyc.org/articulo. oa?id=647/64770116

Salas-Bravo S., González-Árias, M., Araya-Piñones, M., Valencia-Jiménez, M. \& OyarceCortes, S. (2017). Uso del Test de Rendimiento Continuo de Conners para diferenciar niños normales y con TDAH en Chile. Terapia Psicológica, 35(3), 283-291. http:// dx.doi.org/10.4067/S0718-48082017000300283

Sánchez-Trujillo, D. \& Torres-López, L. C. (2018). Sobrediagnóstico del Trastorno por Déficit de Atención e Hiperactividad: producto contextual o afinidad clínica. Rev Electrónica Psiconex, 10(16), 1-15.https://aprendeenlinea.udea.edu.co/revistas/ index.php/Psyconex/article/view/334748/20790551

Santander, J., Berner, J.E., Contreras, A. M. \& Gómez, T. (2013). Prevalencia de déficit atencional en estudiantes de medicina de la Pontificia Universidad Católica de 
Chile. Rev Chil Neuro-Psiquiat; 51(3), 169-174. https://scielo.conicyt.cl/pdf/rchnp/ v51n3/art02.pdf

Sedó, M. (2004). Test de las cinco cifras: una alternativa multilingüe y no lectora al test de Stroop. Revista Española de Neurología, 38 (9), 824-828. https://doi. org/10.33588/rn.3809.2003545

Sedó, M. (2007). Test de los Cinco Dígitos. Madrid: T.E.A. Ediciones.

Sergeant, J. (2005). Modeling Attention-Deficit/Hyperactivity Disorder: A Critical Appraisal of the Cognitive-Energetic Model. Biological Psychiatryc, 57(11), 1248-1255. https://doi.org/10.1016/j.biopsych.2004.09.010

Serón, T. \& Rodríguez, L. (2017). Trastorno por déficit atencional e hiperactividad (TDAH) y su relación con el síndrome de apnea obstructiva del sueño (SAOS) en pediatría. Rev. Ped. Elec., 14(2), 22-26. https://www.revistapediatria.cl/volumenes/2017/ vol14num2/5.html

Servera, M. \& Llabrés, J. (2004). Tarea de Atención Sostenida en la Infancia. Illes Balears: TEA ediciones, S.A.

Sims, D., Purpura, D. \& Lonigan, C. (2012). The Relation Between Inattentive and Hyperactive/Impulsive Behaviors and Early Mathematics Skills. Journal of Attention Disorders, 20(8), 704-714. https://doi.org/ 10.1177/1087054712464390

Štuhec, M., Švab, V., \& Locatelli, I. (2015). Prevalence and incidence of attention-deficit/hyperactivity disorder in Slovenian children and adolescents: a database study from a national perspective. Croatian Medical Journal, 56(2), 159-165. https://doi. org/10.3325/cmj.2015.56.159

Thurstone, L. \& Yela, M. (2012). Test de Percepción de Diferencias CARAS-R. Madrid: TEA Ediciones.

Tirapu-Ustárroz, J., García-Molina, A., Luna-Lario, P. \& Periáñez, J. A. (2012). Evaluación de las funciones ejecutivas. In J. Tirapu-Ustárroz; A. García-Molina; M. Ríos-Lago; A. Ardila (Eds.). Neuropsicología de la corteza prefrontal y las funciones ejecutivas. Barcelona: Viguera: 197-222. https://dialnet.unirioja.es/servlet/articulo?codigo $=4694888$

Ulloa-Flores, R. E. (2009). Cuestionario para Escolares y Adolescentes Latinoamericanos con Trastorno por Déficit de Atención con Hiperactividad (CEAL-TDAH). Validez y confiabilidad temporal. Salud Mental; Vol. 32, Supl. 1: 63-68. https:// www.medigraphic.com/pdfs/salmen/sam-2009/sams091g.pdf

Valdivieso, A., Cornejo, A. \& Sánchez, M. (2000). Tratamiento del síndrome de déficit atencional (SDA) en niños: evaluación de la moclobemida, una alternativa no psicoestimulante. Revista Chilena de Neuro-Psiquiatría, 38(1), 7-14. http://dx.doi. org/10.4067/S0717-92272000000100002

Wolraich, M.L, Wolraich, M., Mckeown, R., Visser, S., Cuffe, S., Neas, B., Geryk, L., Doffing, M., Bottai, M., Abramwitz, A., Beck, L., Holbrook, J. y Danielson, M. (2014). The Prevalence of ADHD: Its Diagnosis and Treatment in Four School Districts Across Two States. Journal of Attention Disorders, 18, 563-575. https://doi. org/10.1177/1087054712453169

Young, S., \& Gudjonsson, G. H. (2005). Neuropsychological correlates of the YAQ-S and YAQ-I self- and informant-reported ADHD symptomatology, emotional and social problems and delinquent behaviour. British Journal of Clinical Psychology, 44(1), 47-57. https://doi.org/10.1348/014466504X197769.

Esta obra está bajo: Creative commons attribution 4.0 international license. El beneficiario de la licencia tiene el derecho de copiar, distribuir, exhibir y representar la obra y hacer obras derivadas siempre y cuando reconozca y cite la obra de la forma especificada por el autor o el licenciante.

\section{(cc) BY}

